\title{
El fraude académico: Un análisis de estudio cualitativo del alumnado en la Facultad de Medicina de la Universidad de Antioquia
}

\section{The Academic fraud in the university training process: An analysis of qualitative study in the Faculty of Medicine of the University of Antioquia}

Germaín Campo-Acosta

Universidad de Antioquia, Medellín, Colombia

\begin{abstract}
Resumen
Objetivo: Develar los significados que emergieron de los estudiantes sobre la práctica del fraude académico, como acto presente durante la formación académica. Método: Este estudio se abordó mediante el paradigma cualitativo con enfoque hermenéutico y utilizando como metodología la Teoría Fundada, dentro del marco conceptual del interaccionismo simbólico. Como herramienta de recolección de datos se utilizó una entrevista semiestructurada a profundidad que permitió el análisis de las visiones, creencias, valores e ideologías que se produjeron entre los entrevistados. Resultados: El estudio mostró que, para los estudiantes, el fraude es considerado como una acción válida y consentida dentro del proceso enseñanza aprendizaje, donde el imperativo categórico es subordinado a la vía fácil, práctica o necesaria para conseguir los objetivos académicos. Discusión y Conclusiones: Los significados que se tejen en los estudiantes del grupo de estudio alrededor del fraude, nos muestran que se ha elaborado un constructo conceptual donde el fraude es una opción válida, cimentada sobre unos valores basados en las necesidades individuales que dejan de lado los códigos deontológicos y de esta manera se convive con él sin mayores reflexiones.
\end{abstract}

Palabras clave: Fraude académico, deshonestidad académica, teoría fundadamentada, interaccionismo simbólico.

\begin{abstract}
Objective: Revealing the meanings that emerged from the students about the practice of academic fraud, as an existing act during the academic preparation. Method: This study was tackled through the qualitative paradigm with a hermeneutical approach, using the Founded Theory as a methodology within the conceptual framework of symbolic interactionism. An indepth semi-structured survey was used as a data collection tool that allowed the analysis of the visions, beliefs, values, and ideologies produced among the interviewees. Results: The study showed that for students, fraud is considered a valid and consensual action within the teachinglearning process, where the categorical imperative is subordinated to the simple, practical, or necessary path to achieve the academic objectives. Discussion y Conclusion: The meanings that are manufactured in students around the concept of fraud show us a well-elaborated idea where fraud is accepted as a valid option, based on values centered on individual needs that leave deontological codes aside, in this way you can live with him without further reflection.
\end{abstract}

Open Access:

ISSN: $0124-2121$ E-ISSN: $2665-2420$

Editor: Dhayana Fernández Matos

Keywords: academic fraud, academic dishonesty, grounded theory, symbolic interactionism.

Cómo citar este artículo (APA): Campo-Acosta, G. (2021). El fraude académico: Un análisis de estudio cualitativo del alumnado en la Facultad de Medicina de la Universidad de Antioquia. Educación y Humanismo, 23(41), 182-204. https://doi.org/10.17081/eduhum.23.41.4113 


\section{Introducción}

El sistema educativo basa su eficacia y la promoción de los estudiantes en la evaluación como método de medición del logro de los objetivos planteados. La evaluación, de esta manera, se convierte en un determinante sistemático del mérito y el avance académico del individuo, que mostrará el logro de los objetivos propuestos cuando evidencie que ha superado las pruebas impuestas por los docentes mediante diferentes herramientas, que en la gran mayoría de casos corresponden a exámenes escritos (Pardo, Rodríguez-Casals, 2019).

Bunn, Caudill y Gropper (1992) consideran que el fraude académico es, "cualquier comportamiento no lícito en un estudiante universitario, en torno a exámenes o a trabajos escritos, realizados como requisito para superar una asignatura", pero en realidad es difícil establecer una definición única de fraude académico, ya que en últimas su conceptualización depende de cada uno de los reglamentos estudiantiles de las instituciones educativas, en un ejercicio de autonomía institucional (Bunn, Caudill y Gropper, 1992; Comas, Sureda, Casero y Morey, 2011).

El fraude o trampa en la universidad no supone solo la obtención de una nota no merecida, va más allá de la correspondencia "Mérito vs Nota", supone la cualificación de un profesional que puede poner en riesgo la integridad física, psíquica o financiera de un grupo de personas. El edificio o el puente construido con error de cálculo se pueden derrumbar, el médico mal fundamentado puede errar en su diagnóstico o tratamiento y el ingeniero financiero con un mal cálculo puede provocar el fracaso financiero (Wiesenfeld y Jaramillo, 2011).

Pero no es solo la práctica errónea por parte del profesional sin las competencias adecuadas que la universidad ha certificado con base en las notas obtenidas mediante la trampa, está también la cultura de la mentira y "el todo vale" convertida en una práctica aceptada como medio de escalar socialmente, sacando provecho personal a costa de lo que sea, convirtiéndonos en una sociedad fracasada, como lo expresa el autor Mario González (Mora, 2015).

La trampa en la educación por parte de los estudiantes tiene grandes consecuencias en su desempeño y en la economía de un país. La experiencia nos ha mostrado que esta práctica que vulnera principios éticos es fácilmente trasladada a los sitios de desempeño profesional. (Mejía-Sabogal et al., 2019) Igualmente se ve cómo los países con índices altos de corrupción muestran una relación directa con la práctica del fraude universitario y viceversa (Ayala-Gaytán y Quintanilla-Domínguez, 2014). 
El fraude académico es una práctica que no escapa a la Facultad de Medicina de la Universidad de Antioquia (UDEA). Un estudio realizado en 4 universidades colombianas de alto prestigio, donde se aplicaron entrevistas directas a más de 3300 estudiantes entre 2003 y 2013, mostró que más del $90 \%$ de los encuestados admitió haber cometido fraude académico y que esta proporción de estudiantes que admitieron el fraude no vario durante la década del estudio. Este estudio al igual que otros realizados dentro y fuera del país corresponden a estudios de prevalencia, por lo que hacen falta estudios específicos que den cuenta de una caracterización más profunda del tema, que permita una posterior intervención para producir un cambio en la práctica del fenómeno. (Martinez y Ramírez, 2018)

Este trabajo pretende dar respuesta a la falta de comprensión del fenómeno de la trampa por parte de los estudiantes en la Facultad de Medicina, teniendo en cuenta las interacciones subjetivas que se construyen entre ellos, para la formación de los significados alrededor del fraude académico. Todo esto de la mano de aspectos como la percepción de la moral institucional (compañeros, profesores y directivos), los grados de severidad y efectividad de las sanciones, la actitud de los profesores en el aula de clase, los mensajes que se trasmiten, lo que se dice y lo que se calla sobre las conductas fraudulentas.

\section{Antecedentes}

En Estados Unidos y Europa existen estudios al respecto, diferente a Colombia o incluso Latinoamérica, donde no son muchos los trabajos e intervenciones que se ocupen del fenómeno del fraude académico (FA). Las investigaciones desarrolladas abarcan los temas de: prevalencia y profundidad del fenómeno; causas; perfil de los infractores y detección e intervención del fenómeno (Fernández y Ordóñez, 2004).

El plagio como uno de los mecanismos del fraude académico, ha sido estudiado aparte, su incidencia es alta y se le vincula al desarrollo tecnológico. Al igual que las demás formas de fraude, se le considera una práctica deshonesta, en contra vía con la formación integral, instigadora de actos corruptos, no promueve el pensamiento crítico y por tanto obstaculiza el proceso enseñanza aprendizaje (Ramírez, 2019).

Los objetivos de las investigaciones publicadas sobre el tema abarcan varios aspectos, entre los que se destacan los encaminados a identificar la percepción que tienen los estudiantes de lo que se considera fraude y los motivos por los cuales los llevan a cabo (Martinez-Gomez, Borjas y Andrade, 2015; Teodorescu et al., 2007). Otros investigan sobre las causas sociodemográficas que pueden estar relacionadas con la práctica del FA, donde se mira la relación que puede haber entre el fraude y las características generales como género, nacionalidad, etnia o situación social de los estudiantes (Cronan, Mullins y Douglas, 2015), (Beasley, 2016). 
Existen también estudios que buscan causas más exógenas y apuntan a identificar la incidencia que pueda tener en la presencia del FA, el papel de las instituciones a través de sus reglamentos sobre la evaluación, las normas de derechos y deberes del alumnado así como los códigos éticos y disciplinares (Sureda-Negre, Comas-Forgas y Oliver-Trobat, 2015).

Otros tipos de estudios se han orientado hacia la identificación, sanción y control punitivo del fraude académico con pobres resultados. Ya que ha sido muy difícil llegar a conclusiones férreas aún con panel de expertos, sobre cómo dimensionar la gravedad y repercusión de los comportamientos deshonestos en los que puede incurrir el alumnado. Esta Situación debida probablemente a la falta de trabajos investigativos que aborden las conductas fraudulentas de los estudiantes (Sureda-Negre, Cerdá-Navarro, Calvo-Sastre y Forgas, 2020).

En España y otros países europeos se ha indagado sobre la percepción de los estudiantes ante políticas institucionales y de aula frente al plagio académico. Se preguntó sobre la existencia y eficacia de la normativa ética reguladora y de la actuación del profesorado en las aulas. Los estudiantes valoraron de manera negativa las causas motivadoras del plagio y de forma más positiva las actuaciones del docente en el aula (Castro, Espiñeira-Bellón, Losada-Puente y Pérez, 2019).

Al margen de las investigaciones anteriores que son estudios sociales orientados hacia las percepciones de los estudiantes para dilucidar los motivos por los cuales se presenta el FA desde lo comportamental, existe un estudio en China que busca la relación entre las características biológicas y la conducta violatoria de la integridad académica desde una perspectiva meramente mecanicista, donde las percepciones, interacciones, significados y valores éticos no son considerados. En este estudio se muestra la relación entre las variantes genéticas de la triptófano hidroxinasa y la conducta violatoria de la integridad académica (Shen et al., 2016).

En Antioquía, en el año 2013, en el marco del programa de desarrollo social "Antioquia legal", se realizó una campaña para identificar y abordar el fenómeno del fraude en las universidades; se encuestaron 5944 estudiantes de 15 instituciones públicas y privadas del departamento donde se obtuvieron las siguientes cifras: $80 \%$ admitieron haber dejado que un compañero le copie en un examen; $56 \%$ dijeron que han copiado respuestas de un compañero; el $9 \%$ confesó haber presentado un certificado médico falso para justificar una inasistencia; más del $75 \%$ de los alumnos aceptaron haber cometido algún tipo de fraude; $11 \%$ mostró como de su autoría un trabajo que copió de la internet y $3.2 \%$ realizó un examen por un compañero (Martinez y Ramírez, 2018).

Las publicaciones existentes hacen referencia principalmente a la frecuencia y los motivos que aducen los estudiantes para realizar el fraude, pero no se cuestionan lo que significa para estos desde lo ético, social y académico. Con este trabajo se pretende precisamente un abordaje en profundidad enmarcado en las teorías del interaccionismo simbólico y la 
formación de los signos y significados. El pensamiento complejo también se abordó, pero solo desde una perspectiva de fondo sin profundización teórica. Estos enfoques nos permitieron comprender la manera como se presenta el fenómeno del fraude en su cotidianidad académica en la Facultad de Medicina de la UDEA.

\section{Contexto social}

El fraude académico (FA) constituye un fenómeno no deseado en las instituciones de educación superior, sin embargo, se evidencia que su práctica se presenta con bastante regularidad en los ámbitos académicos universitarios de Europa, Estados Unidos y América Latina. Diferentes estudios revelan que más de $80 \%$ de los estudiantes han realizado FA alguna vez durante el curso de su carrera (Beléndez-Vázquez et al., 2011), (Ceballos-Villada, 2011). Igualmente en Colombia se muestran estudios que reflejan datos similares en la práctica del fraude por parte de los estudiantes (Ordóñez; Mejía; Castellanos, 2006). Esto pone en relieve la importancia que merece la caracterización del fenómeno por parte de la comunidad académica.

El Internet por ser la fuente principal o única de consulta a la hora de realizar trabajos, se constituye en la principal fuente de plagio académico, seguido de la copia de textos escritos o de trabajo de los compañeros (Gama et al., 2013).

Los motivos para estas prácticas son diversos y abarcan los que tienen que ver con la alta competitividad, la ignorancia de las normas, la presión social, el acceso laboral, convenciones que representan lo que es lícito o no, falta de reglas para mantener y hacerlas cumplir, y las deficiencias en la mecanismos para detectar y hacer frente a las infracciones (Eckstein, 2003).

Un estudio reciente muestra que más del $90 \%$ de los participantes admiten que las causas obedecen a de estudio, dedicación, interés y ausencias a las actividades de enseñanza. Sin embargo justifican la práctica del fraude en la falta de tiempo para estudiar, el no dominio de los métodos de estudio eficientes, la ignorancia del daño que este hecho puede causar y la presencia de problemas personales y familiares que presionan al estudiante. También aludieron causas inherentes a la institución como son, la falta de rigor de los maestros y el personal con respecto a las medidas organizativas en las pruebas y la falta de calidad en las actividades de enseñanza (Chipombela, Pereira y Calivangue, 2020).

El desarrollo de la cultura en Colombia ha adquirido unas características propicias para la práctica de lo ilícito que fácilmente se traslada a los estudiantes dentro del desarrollo de sus actividades académicas. Luis Fernando Duque, al describir la cultura del país, muestra las tipologías sustantivas del colombiano de hoy, de quien dice que estos siguen reglas de comportamientos basadas en un desequilibrio entre lo privado y lo público; entre lo individual y lo colectivo; que hace que desarrollen un espíritu emprendedor, capaz de salir 
de manera individual de situaciones difíciles, pero que a la vez lo convierten en una persona con imposibilidad de crear relaciones duraderas (Duque, Toro y Montoya, 2010).

En esta cultura que forma el colombiano de hoy, donde lo individual prima sobre lo colectivo con un determinismo muy claro, donde se está dispuesto a aprovechar todas las oportunidades para beneficio propio, aludiendo a la cultura del "todo vale", reforzada por la falta de una institucionalidad que regule, enseñe y sancione (Duque, Toro y Montoya, 2010).

Estas características redundan en una cultura rentista y de ilegalidad con gran desprecio por lo social o lo público, donde el quebrantamiento de la norma y la poca o nula valoración de los derechos de los demás son validados como medio de sobrevivencia. En este contexto los atajos, las trampas y el plagio se convierten en prácticas rutinarias que pierden la condición de ilegales y se practican rutinariamente bajo el concepto de "viveza" (Duque, Toro y Montoya, 2010).

El narcotráfico es un claro ejemplo de esta cultura, donde se remplazan los valores fundamentales por el todo vale. El dinero, no importa de dónde provenga, se vuelve el rasero de medición más que los méritos o los logros por esfuerzo propio. La cultura predominante valora los "atajos", la "trampa" y hacemos apología de estas en el cine (Mejía-Quintana, 2018).

\section{El interaccionismo simbólico y pensamiento complejo}

El interaccionismo simbólico junto con la teoría fundada ha sido empleado para investigaciones en el campo de la sociología, la psicología y hace unos pocos años en el campo de la educación. El interaccionismo simbólico permite una apreciación en profundidad sobre los significados que se forman los estudiantes en las interacciones sociales, y que los llevan a conducirse dentro y fuera del aula. Por otra parte, la teoría fundada, como diseño metodológico que ayuda a la sistematización ofrece una herramienta ampliamente confiable para el análisis de datos en las investigaciones cualitativas (Suárez, 2021).

Generar teoría resulta muy útil para entender e intervenir un fenómeno cultural, y para esto es necesario conocer de ante mano la dinámica de la sociedad que se quiere modificar. No se pueden generar los cambios esperados si no se conoce que actores intervienen, que fuerzas predominan cual es la simbología que se genera de la interacción social, que poderes emergen, quienes los desafían y quienes son excluidos. Es necesario tener una perspectiva amplia de estos aspectos para lograr impacto (Vich, 2019).

La manera como actuamos, como nos percibimos e interpretamos la realidad obedece a lo que autores como George Herbert Mead y Herbert Blumer, entre otros, han definido como interaccionismo simbólico. Ellos explican cómo en la interacción social se construyen significados que van a determinar la percepción del entorno, la conducta y la 
conciencia del "Yo". Entendiendo el yo como el cuadro que me formo de mí mismo y que proviene de las reacciones que tienen los demás a partir de mí actuar (Blumer y Alonso, 1982).

Los significados que emergen de las interacciones sociales dan sentido a la conducta, es en el interaccionismo donde radica la esencia humana ya que la conciencia de la propia existencia se forma en la interacción. El sentido del "Yo" adquiere realidad cuando las personas se relacionan y son reconocidas por el otro. De esta forma los significados productos de la interacción devienen la realidad del sujeto mediante procesos intersubjetivos que producen nuevas realidades modificando la conducta (Blumer y Alonso, 1982).

El interaccionismo simbólico es simultáneamente una corriente teórica y un marco metodológico en ciencias sociales, ya que trata de comprender el proceso de asignación de símbolos con significado al lenguaje hablado o escrito y el comportamiento en la interacción social; se encuentra clasificado dentro de las corrientes sociológicas interpretativas como una de las más exitosas por muchos autores (Suárez, 2021).

Este trabajo se propuso profundizar en los significados que emergen en los estudiantes de la Facultad de Medicina de la UDEA sobre el concepto de fraude universitario y su relación con la práctica del fenómeno, concibiendo "significado" en el sentido que Lev Semionovich Vygotsky propone: "...entiende los significados como aquellas representaciones que construye el sujeto por medio del uso de signos....,inicialmente surgen en la relación y luego en el pensamiento" (Mendoza, Ramos, Ortiz y Jaramillo, 2010).

Para Vygotsky los signos son eventos o sucesos que se intercalan entre el estímulo y respuesta, que, en el caso del ser humano diferente a las demás especies, ya que se construye de manera activa, es decir, no corresponde a una respuesta condicionada como cuando un perro responde a una campana, en las personas los signos son herramientas creadas para regular su propio comportamiento y el de los demás. Esto lo define Vygotsky como: "estímulos-medio artificiales introducidos por el hombre" (Gutiérrez, Ball y Márquez, 2008).

El pensamiento complejo, como se denomina, es una concepción de orden profundo no superficial, que presenta la formación de las ideas y la conducta derivada de ellas desde una perspectiva holística, de un proceso finamente elaborado donde intervienen e interactúan varios factores de estudio antropológico, allí es imposible separar las relaciones en la praxis de cuatro factores que son a la vez sistemáticos, complementarios y antagónicos: el sistema genético, el cerebro, el sistema sociocultural, y el ecosistema (Arancibia, 2010).

El pensamiento complejo nos invita a cuestionarnos la interpretación de la realidad concebida como el conjunto de hechos concluidos a partir del conocimiento parcelado de las ciencias, la cual si bien es cierto, ha producido grandes adelantos tecnológicos y saltos 
de progreso, ha ocasionado también que se pierda de vista la trama del mundo real, su tejido en conjunto, a partir del interaccionismo y la complementación, "complexus" (Hernández, 2013)

\section{Método}

La base epistemológica para la ejecución del proyecto es la teoría fundada o fundamentada, en esta se consideraron citas bibliográficas de los autores Strauss y Corbin (2002), proponentes del modelo, y de otros entre los que se encuentran autores como Giménez (2007) quien ha trabajado el modelo en áreas diferentes a las sociales como la administración.

Para este estudio se recopilaron los datos a través de una entrevista semiestructurada aplicada a 10 estudiantes, de los cuales 6 pertenecen al pregrado de Medicina y 4 al pregrado de Instrumentación Quirúrgica en la Facultad de Medicina de la UDEA; estas se ejecutaron presencialmente por uno de los investigadores.

En el ejercicio se obtuvieron 309 citas o unidades de análisis de las 10 entrevistas y se procedió con la codificación abierta de la cual emergieron 281 códigos. Posteriormente, se procedió a la categorización, donde los códigos obtenidos se agruparon de acuerdo con una abstracción más elevada, de esta manera se obtuvieron ideas más grandes que fueron dando la explicación de los fenómenos emergentes en los datos. Del ejercicio resultaron 26 categorías, estas subcategorías se relacionaron entre sí y de esta manera se construyeron fenómenos más explicativos de las diferentes teorías, los memos (notas explicativas de la codificación) obtenidos mediante la codificación abierta, se trajeron durante el análisis de las categorías y subcategorías, estas notas explicativas, ayudaron a dar cuerpo al mapa de los fenómenos emergentes. La codificación, categorización, análisis y la emergencia de los fenómenos se obtuvieron siguiendo los pasos de la teoría fundada planteados por los sociólogos Barney Glaser y Anselm Strauss (Strauss y Corbin, 2002)

En esta etapa del análisis, como en las demás, no se perdieron de vista los datos originales, esto fue posible de manera muy práctica con el programa de análisis utilizado: Atlas Ti 7.5.4, el cual permitió a partir de cualquier código importar las citas relacionadas del documento original y así ir vinculando las diferentes categorías, recoger el sentido real emanado de los datos y no perderse en conceptos propios que se alejen de ellos.

Las relaciones de las categorías se realizaron mediante diagramas que muestran las interconexiones de las diferentes categorías, donde a partir de una categoría central se relacionaron las otras. Esta diagramación permitió un análisis visual que facilitó la inclusión de todos los aspectos de la red semántica (Strauss y Corbin, 2002). A continuación se muestra un ejemplo de diagrama, donde se relacionan las diferentes categorías resultantes. 
Figura 1.

Relación de categorías, red semántica para el análisis

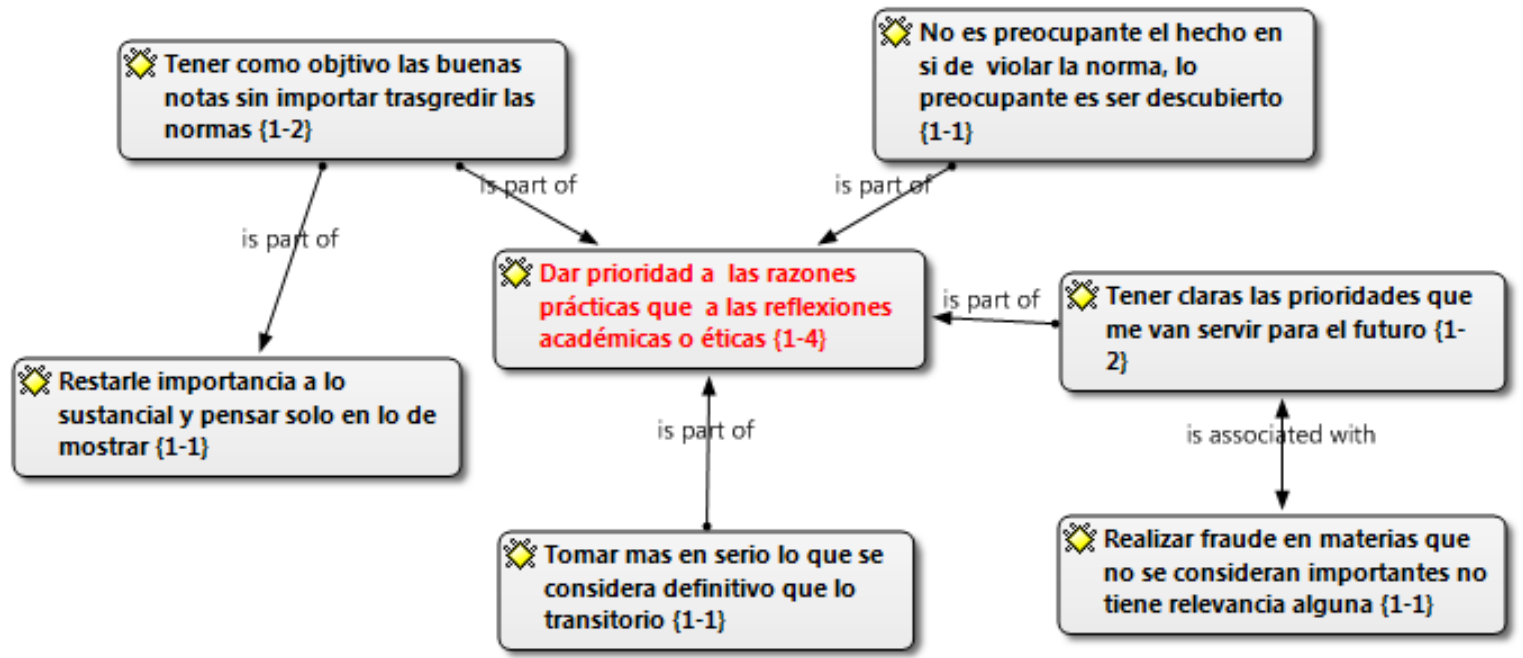

Fuente: Elaboración propia (2021)

Relación de las categorías resultantes de la diagramación de la red semántica.

Existe una aceptación social de las conductas fraudulentas que hace que el fraude se convierta en un fenómeno invisible.

Se evidencia una normalización de la conducta del fraude; su frecuencia es tal que termina siendo parte del paisaje, del día a día, se convive con él con pocas críticas sin mayores reflexiones, de alguna forma termina siendo legitimado por algunos y soportado por otros. La práctica del fraude se convierte en algo normal que es compartido entre amigos, institucionalizado.

Respuesta de estudiante: "Exactamente, si se ejecuta un fraude es normal, sea porque se necesitaba, sea las cuestiones que sea, el estudiantado como tal lo maneja como algo corriente".

Pregunta ¿No hay vergüenza, no hay pena frente al hecho de cometer fraude?

Respuesta de estudiante: "No para nada, no hay vergüenza ni pena". (Fragmento de entrevista Atlas Ti, Proyecto Fraude académico, 2016, cita 5:6)

Sin embargo, hay un sentimiento interior de lo incorrecto de las acciones, que también se acostumbran a compartir: 
Respuesta de estudiante "Entre nosotros los estudiantes lo vemos como algo común, pero en realidad uno lo nota y no dice nada, uno ve mucho cuando está en la silla, uno hasta ve más que el profesor y uno ve que está mal hecho, pero todos los estudiantes lo toman muy normal, una práctica cotidiana en el área de estudio, tanto para el que lo práctica como para el que no, aunque yo sé que en el ámbito general está mal hecho, como decía anteriormente, uno mismo se justifica" (Fragmento de entrevista Atlas Ti, Proyecto Fraude académico, 2016, cita 7:5).

Las emociones que tenemos, contienen un sustrato cognitivo y no meramente sensitivo, la estructura de las emociones está constituida por creencia, juicios o cogniciones que constituyen el núcleo de la moral y potencializan las acciones. Desde esta teoría podemos deducir que las acciones no obedecen únicamente a la razón y que las emociones no se quedan en la escala sensitiva si no que determinan la conducta (Camps, 2012).

Lo pragmático se sobrepone a lo ético.

Lo que prima es lo práctico, lo inmediato, los resultados, más no los procesos involucrados desde la perspectiva moral y ética. Se resta importancia a lo que no sea socialmente destacable, convirtiendo en objetivo principal solo la obtención de buenas notas, y en todo este proceso no tiene relevancia si se realiza fraude, lo importante es no ser descubierto: "es el caso de uno realizar una prueba y ver compañeros entre sí gestualizando, haciendo señas, entonces si es algo que se toca, pero a la vez es algo como que siempre y cuando al estudiante no se le descubra no es algo que le preocupe demasiado"(Fragmento de entrevista Atlas Ti, Proyecto Fraude Académico, 2016 cita 5:26).

Existe una clasificación de las materias y así mismo el fraude es jerarquizado; la gravedad de este depende de las materias donde se efectúe. Se juzga el fraude por su impacto en el desarrollo académico más no por sus consecuencias éticas. "Porque yo pienso que hay estudiantes que jerarquizamos las materias, hay unas materias mucho más importantes, voy a dar un ejemplo, uno se fija, no sé, inglés, yo creo que uno miraría que hacer fraude en esa materia no es como tan grave, el trabajo de grado si es grave porque es algo que se va a publicar. (Fragmento de entrevista Atlas Ti, Proyecto Fraude académico, 2016, cita $6: 14)$

Camps (2007) en su libro "La excelencia de las profesiones sanitarias" permite reflexionar sobre lo que se considera un éxito profesional. En ese texto explica la importancia del conocimiento técnico al margen de los códigos deontológicos. La fama y el dinero se convierten así en el único rasero para medir lo que es considerado una buena carrera, sin reparar que para llegar a ella se hubiese tenido que imponer lo particular sobre el bien general (Camps, 2007).

Hay una promoción de estudiantes sin las debidas competencias gracias al fraude académico. 
Una preocupación manifiesta de los estudiantes es la promoción indebida de compañeros que no tienen las competencias necesarias, pero mediante el fraude logran graduarse y acceder a posiciones laborales mejores que muchos estudiantes que no lo practican.

Esta situación significa para ellos un perjuicio social manifiesto, traducido en profesionales no aptos que van a atender pacientes sin los debidos conocimientos, además del deterioro de la imagen de la universidad, sobre la cual ellos consideran que ha decaído por la promoción de estudiantes no aptos que han aprobado sus materias valiéndose del fraude académico.

Respuesta de estudiante "Si, sobre todo porque la facultad no es muy estricta en la promoción, o sea me refiero, por decirlo así, en la evaluación. Entonces muchas veces, y por ejemplo en el pregrado se ve que pasan compañeros que definitivamente no adquirieron los conocimientos que se debieron adquirir, entonces en una parte ya práctica donde vamos a otros hospitales y nos preguntan, esos compañeros no saben y hacen quedar mal a la facultad; y en parte eso se discute porque nos están juzgando a todos a partir de eso, y cuando empieza uno hablar de esto, eso va a salir y afuera se van a dar cuenta que en la facultad no se hace todo tan bien como era el imaginario hace algún tiempo, que para mí se ha ido perdiendo ese imaginario" (Fragmento de entrevista Atlas Ti, Proyecto Fraude Académico, 2016 cita 10:9).

Estas ideas van de la mano con algunos estudios sobre el tema que dan cuenta de cómo la idoneidad profesional es defectuosa cuando se evidencia la práctica del fraude durante los estudios universitarios (Agud, 2014). Sin embargo, hay quienes manifiestan que no en todas las ocasiones los estudiantes que realizan fraude terminarán siendo malos profesionales, pues existen algunos estudiantes que siendo buenos académicamente realizan fraude no solo por el logro de una promoción, sino por la necesidad de ser reconocidos como los mejores.

El proceso de control y la censura hacia el fraude es mínima e incentiva su práctica.

Durante las pruebas escritas los estudiantes evidencian la utilización de dispositivos móviles por parte de sus compañeros para efectuar fraude, esto junto a la tenencia de parciales antes de las pruebas, son referentes para que los estudiantes reafirmen que, las medidas de control y las sanciones, no son importantes para los docentes ni para la institución: "P: ¿es posible manipular un celular durante un parcial? R: Sí, o sea por reglamento no se debe hacer, pero cuando es un examen con grupos inclusive de ciento veinte, ciento cincuenta estudiantes y el que está cuidando es incluso un solo profesor, uno escucha el mensaje y alcanza a ver compañeros que se inventan la forma de poder responder los mensajes" (Fragmento de entrevista Atlas Ti, Proyecto Fraude académico 2016, cita 10:4). 
Lo anterior hace que los estudiantes que tienen una posición crítica frente a la trampa, no se sientan seguros ni apoyados para denunciar una acción fraudulenta de los compañeros, ya que no hay credibilidad frente a los procesos sancionatorios por parte de los docentes o la institución. "También siente uno (o he percibido) que cuando denuncian no se le da tanta relevancia al caso, porque a mí también me tocó que un compañero se robó prácticamente un examen en una clase que no se pudo hacer y lo rotó, entonces al otro día se dio la clase y el profesor sabía que se habían robado el examen, pero sencillamente lo que hizo fue cambiar unas preguntas y no se le dio trascendencia a un hecho tan grave. Entonces yo siento que falta un poco también de ser más estrictos con el fraude"(Fragmento de entrevista Atlas Ti, Proyecto Fraude académico 2016, cita 10:18).

Estar inmersos en un ambiente académico de justificación y aceptación del fraude.

Se evidencia la decisión de tomar ventaja cada vez que se presente la oportunidad, infringir las normas aduciendo necesidad, inmadurez, porque constituye el menor esfuerzo o simplemente por considerar que es una constante que no tiene solución, que todo el mundo lo hace y por lo tanto quien no lo haga quedará en desventaja: "Hay profesores que uno dice nunca me han pillado, entonces uno lo sigue haciendo uno ya sabe con qué profesor puede y con qué profesor no". "Pues, a ver, es algo que nunca va a dejar de existir porque el fraude siempre se va a ver ya sea poco o sea mucho, siempre va a estar" (Fragmento de entrevista Atlas ti, Proyecto Fraude Académico, 2016, citas 1:74, 8:22).

Nuestra sociedad concibe un imaginario de hombre individualista, incapaz de confiar y ser confiable para el otro, que considera el atajo y la trampa como algo integrado al diario vivir y sin posibilidad de cambio donde esta falta de confianza se extiende a los organismos de control (Duque, Toro y Montoya, 2010). De esta manera, la concepción del fraude como algo generalizado y sin solución, hace parte del hecho de la no formulación de denuncias oficiales frente a este, ya que no hay un ambiente de confianza de cara a la solución del problema.

Se minimiza el fraude como una cuestión momentánea que solo se quedará en el ámbito universitario y que no se traslada a otros aspectos de la vida. Si se logran los objetivos de aprendizaje, la consecución de una nota, o si se considera que faltan garantías académicas, el fraude será una opción válida que no merece reflexión alguna.

Respuesta de estudiante: "puntualizar, creo que es muy importante no mirar esa situación aislada como es el fraude si no que hay unas circunstancias alrededor de ella que la llevan pues a que se realice, principalmente el tipo de evaluaciones que se hacen, el tiempo que se le den para la preparación de los exámenes, porque hay muchas veces en las que uno se ve con muy poco tiempo, entonces uno no alcanza a revisar todos los temas o pues todo lo que tiene estudiar" (Fragmento de entrevista Atlas ti, Proyecto Fraude Académico, 2016, citas 4:49). 
Se considera al fraude como una opción válida y justificada sin sentimientos de dolo.

Se concibe el fraude como una opción válida y lícita como medio para lograr los objetivos. Los valores éticos no son considerados puesto que prevalece la necesidad por lo cual se justifica el fraude aduciendo razones de justicia, de esfuerzo, de importancia de los temas, de las incapacidades de quien lo efectúa o simplemente porque son oportunidades que no se deben desaprovechar.

Respuesta de estudiante: "pero si uno necesita una nota en este caso lamentablemente tengo que decirlo uno tiene que recurrir a ayudarse, porque es que la universidad lo limita mucho a uno en eso, porque si uno pierde una materia se atrasa un semestre, porque acá muchas son prerrequisitos de otras o correquisitos, entonces uno decir: no, yo no me voy a atrasar por esta materia sabiendo que necesito cierta nota, entonces lamentablemente en ocasiones pues ciertas circunstancias lo llevarían a uno a eso" (Fragmento de entrevista Atlas Ti, proyecto Fraude Académico 2016, cita 4:50).

En este contexto, aunque hay entendimiento en que se está violando la norma, existe un sentimiento interno de que no se transgrede lo ético, ya que hay razones de peso para llevar a cabo las acciones fraudulentas.

Respuesta de estudiante: "No, yo no creo que uno catalogue a una persona solamente porque cometió un fraude". "Pues uno sabe que hay gente que igualmente estudia y que tiene sus ayudas didácticas por decirlo así, pero ahí si no sería tanto por necesidad de la nota sino que pues no se ahí si no sería otra forma así de estudiar porque uno sabe que esas personas estudian los temas, pero que pueden tener algún examen, alguna otra foto ahí que les pueda ayudar a sacar una mejor nota, pues que independientemente que tengan esa ayuda o esas fotos o ese examen o lo que sea, uno piensa que ellos también podrían sacar una buena nota"(Fragmento de entrevista Atlas Ti, proyecto Fraude Académico 2016, citas 6:10, 3:5).

Existe una responsabilidad institucional en la presencia del fraude académico referente al control y sanción. Se evidencia un ambiente de impunidad ante la presencia del fenómeno.

Se evidencia falta de medidas tendientes a controlar y sancionar el fraude, el cual contribuye a que los estudiantes se promuevan sin que se les sancione, aun estando identificados:

Respuesta de estudiante: "Específicamente acá en la Facultad de Medicina es frecuente que uno lo ve, sobre todo hay un fenómeno acá en la Facultad que es como un grupo de estudiantes que son muy malos en Medicina, y hay un grupito que cada semestre lo tiene característico y son estudiantes que la verdad son demasiado malos y que todos nos cuestionamos ¿Por qué semestre a semestre pasan?" (Fragmento de entrevista Atlas Ti, Proyecto Fraude Académico, 2016 cita 9:2). 
Entre las medidas de control consideradas están la adecuada selección de los estudiantes teniendo en cuenta también lo actitudinal, ser más estrictos con los procesos disciplinarios, la planeación y ejecución de clases y parciales y un trabajo de concientización desde el inicio a la universidad, ya que los estudiantes llegan con deseos de aprender y con la idea que en la universidad todo se hace bien, pero cuando identifican que hay una cultura de fraude se adhieren a ella.

Respuesta de estudiante: " $Y$ entonces uno empieza a mirar y en las rotaciones así no vayan o no respondan en lo que es, los pasan y les ponen buena nota; en los exámenes cuando llegan la mayoría de ellos ya tienen los exámenes, se los consiguen porque generación a generación se los van pasando y como eso sale generalmente de un banco de preguntas, entonces ellos ya llegan al examen sabiéndose gran parte del examen; ni siquiera se cuestionan el porqué de cada pregunta, si no que de manera automática lo hacen, se ubican siempre de manera estratégica en cada una de las partes y se lo pastelean todo y se lo miran a los otros compañeros" (Fragmento de entrevista Atlas Ti, Proyecto Fraude Académico, 2016 cita 9: 13).

No se perciben acciones concretas y eficaces en contra del fraude académico por parte de los docentes y demás organismos académicos y disciplinarios de la Facultad.

Respuesta de estudiante: "y con los profesores tampoco creo que le den mucho énfasis en eso, ya que ellos nada más citan que si usted hace fraude podría acarrearle problemas disciplinarios, y se va a un proceso disciplinario, pero pues ¿que uno vea que efectúen algún programa o alguna medida contra eso? No, uno no lo ve tan frecuentemente" (Fragmento de entrevista Atlas Ti, Proyecto Fraude académico, 2016, cita: 2:29).

Esto crea un ambiente de aceptación a la violación de la norma, ya que no hay mucho que perder y en cambio sí se obtienen mejores notas con el fraude por que el reglamento estudiantil se queda en el papel.

Respuesta de estudiante: "pero, usted siempre más o menos así, usted siempre ve en todos los exámenes como que se hablan y esas personas lo toman asi: a no es que siempre lo hemos hecho y no nos ha pasado nada, entonces es algo que ya ven como tan natural" (Fragmento de entrevista Atlas Ti, Proyecto Fraude académico, 2016, cita: 4:33).

Por esto, para evitar la frecuencia del fraude, se reclaman acciones sancionatorias más drásticas que sirvan de ejemplo y desestimulen las prácticas ilícitas durante los parciales.

Respuesta de estudiante: "Realmente deberían tomar acciones más drásticas, se comprueba que el estudiante está en fraude y realmente aplicar el reglamento o quitarle el examen y que el estudiante lo pierda, pero acá los profesores son muchas veces muy tranquilos con los exámenes" (Fragmento de entrevista Atlas Ti, Proyecto Fraude académico, 2016, cita: 9:28). 
El fraude académico trasciende el aula, contribuye a la corrupción y a la desigualdad social. (Gráfico 8)

El fraude académico no se queda en el aula, se traslada a la sociedad extendiendo la corrupción. Su afectación es en cadena, iniciando por el mismo estudiante y termina impactando a la sociedad donde ese futuro profesional se desempeñe, ya que las personas que logran graduarse mediante el fraude serán malos profesionales incorporados a una fuerza de trabajo que confía en la idoneidad certificada por una universidad de prestigio.

Respuesta de estudiante: "podría poner unas cositas u obviar otras cosas o incluso los mismos compañeros dicen no revisamos una parte, pero entonces pongámoslo como normal, entonces si se podría decir que es un fraude, pero ya no estaría afectando tanto al estudiante si no al mismo paciente como tal y a sus colegas en un futuro cercano" (Fragmento de entrevista Atlas Ti, Proyecto Fraude académico, 2016, cita: 2:3).

Esto genera una sensación de desigualdad o de injusticia puesto que se identifica que muchos de esos estudiantes que lograron su título por medio del fraude, muchas veces logran mejores puestos que aquellos que lograron su título sin recurrir al fraude. Esto se suma al hecho de acostumbrarse a la trampa y llevarla a todos los ámbitos de desempeño social; se crea la costumbre por el hábito y se vuelve una forma de vida.

Respuesta de estudiante: "Si yo creo que, si es una conducta que se va teniendo como hábito pues que a uno ya no le importa, ya no le da susto por decirlo así, es algo que usted lo toma demasiado normal en la vida yo creo que cualquier otra cosa que usted haga por fuera de la universidad puede hacerlo fácilmente" (Fragmento de entrevista Atlas Ti, Proyecto Fraude Académico, 2016, cita: 3:10).

\section{Resultados}

Del análisis final de los datos emergieron 3 fenómenos principales que resumen la percepción que tienen los estudiantes de la práctica del fraude académico en la Facultad de Medicina de la UDEA:

Fenómeno 1: El fraude como una acción válida y consentida dentro del proceso de aprendizaje, concepto reforzado por las siguientes condiciones:

- Estar inmersos en una cultura de trampa

- Las acciones ilícitas validadas cuando se percibe injusticia

- Escepticismo sobre las políticas sancionatorias

- Clima de impunidad respecto al fraude académico 
Fenómeno 2: El imperativo categórico es subordinado a lo fácil, práctico o necesario como resultado de las siguientes condiciones:

- La cultura del atajo como medio de sobrevivencia

- La necesidad como único dictamen para la conducta. Esto reforzado por:

- Considerar que las necesidades particulares están por encima del bien común

Fenómeno 3: Profesionales sin las debidas competencias, promovidos mediante el fraude académico, acción favorecida por las siguientes condiciones:

- $\quad$ Acceso a los parciales por parte de los estudiantes

- $\quad$ Estrategias de fraude que incluyen el uso de dispositivos móviles

- Falta de control durante las evaluaciones

- $\quad$ Fallas en la evaluación de la práctica

- $\quad$ Elaboración de pruebas que no miden realmente el conocimiento

- Determinación de los estudiantes a promoverse a cualquier costo

\section{Discusiones y conclusiones}

\section{Comportamiento fraudulento}

El comportamiento fraudulento en la Facultad de Medicina de la UDEA, tiene una presencia reconocida por los estudiantes, con características similares en cuanto a motivos, métodos y valoraciones éticas y morales de otros estudios donde han emergido fenómenos parecidos.

Las formas de fraude durante las evaluaciones van desde mirar al compañero, hablar, hasta el uso de dispositivos móviles para mirar y trasmitir información. El modelo evaluativo, el tipo de preguntas la falta de control, la presión social y académicas son aducidas como causas exógenas, mientras que la "vagancia" la procrastinación y la incapacidad académica constituyen las principales causas endógenas que motivan el FA.

El comportamiento fraudulento es practicado por todo tipo de estudiantes, sin diferenciación por sexo o estrato social, muchos de los estudiantes considerados como "buenos" también incurren el fraude por razones de reconocimiento social o el logro de estímulos académicos. 


\section{Representaciones de los estudiantes sobre el FA}

Los significados que se tejen en los estudiantes del grupo de estudio alrededor del fraude, nos muestran que se ha elaborado un constructo conceptual donde el fraude es una opción válida, cimentado sobre unos valores que propenden por las necesidades individuales y dejan de lado los códigos deontológicos.

Se evidencia una normalización de la conducta del fraude, su frecuencia es tal que termina siendo parte del paisaje, del día a día; se convive con él con pocas críticas y sin mayores reflexiones; de alguna forma termina siendo legitimado por algunos y soportado por otros. Las acciones fraudulentas terminan siendo algo compartido entre amigos, institucionalizado, integrado a los códigos de conducta social de los estudiantes.

Pues bien, en estos códigos lo que prima es lo práctico, lo inmediato, los resultados, más no los procesos involucrados. Se resta importancia a lo que no sea de mostrar o transitorio, siendo el objetivo las buenas notas y en todo este proceso no tiene relevancia si se realiza fraude, lo importante es no ser descubierto.

La falta de control y de intervención del fenómeno por parte de los actores universitarios, manda un mensaje de tolerancia que alienta la normalización del fenómeno del fraude. Las sanciones descritas en el reglamento estudiantil no son específicas y están lejos de ser aplicadas. No existe una difusión abierta sobre la existencia del fenómeno, reforzando así el mensaje que los docentes y demás estamentos de la facultad no saben lidiar con el problema.

\section{Sentimientos de los estudiantes hacia el fraude}

Aun cuando en los estudiantes hay juicios de valor que juzgan como incorrecto la práctica del FA, esto no es un obstáculo para dejar de realizarla, los beneficios obtenidos versus las sanciones, unido a la tolerancia general hacia el fraude, hacen que los sentimientos en contra de la práctica del fraude se queden solo en una reflexión pasajera que se deja de lado.

Los estudiantes llegan a la universidad cargados de expectativas altruistas, con el imaginario que, en ese nuevo espacio cultural, que goza de más prestigio que el colegio, las actitudes son más centradas y las normas más estrictas, pero con el tiempo van aprendiendo que no hay tal diferencia y fácilmente se van impregnando de la cultura de la trampa existente, formando así nuevos significados respecto a los conceptos altruistas iniciales.

Los juicios de valor de los estudiantes hacia las conductas fraudulentas, tácitamente se relacionan con el hecho que el fraude permite promover estudiantes incompetentes, pero al margen de esto no hay una censura sentida por los valores éticos y morales que se 
encuentran involucrados en el fenómeno del fraude. Así las cosas, el juicio sigue siendo práctico, ya que lo que se juzga es el hecho de poner en tela de juicio la competencia de los egresados, pero no hay una reflexión moral y ética en profundidad, la preocupación se centra en el desprestigio de la carrera por permitir la promoción de profesionales incompetentes.

\section{Recomendaciones}

Las teorías emergentes de los participantes del estudio nos dan luces para proponer dos líneas de trabajo que pueden contribuir a la disminución del fraude académico: línea punitiva e información y concienciación. Si bien las dos líneas se complementan, en algún momento pueden ser antagónicas, ya que no es apreciable pretender un mandato autónomo con unos códigos punitivos amenazantes. Sin embargo, la naturaleza de nuestra sociedad hace necesario la normalización y la sanción para alcanzar buenos hábitos de conducta social.

\section{Línea punitiva}

Son importantes las acciones administrativas que manden un mensaje de no complacencia con la presencia del fraude académico y la implementación de medidas que lleven a la visualización, control y corrección de las acciones fraudulentas, mediante acciones que involucren a docentes y estudiantes

El reglamento estudiantil debe contener un capítulo especial referente al fraude académico donde se identifiquen plenamente las sanciones a las que pueden ser objeto las personas que trasgredan la norma, con lineamentos claros que indiquen el procedimiento a seguir por parte de los profesores en cada situación.

\section{Información y concienciación}

Esta es la acción más importante que, según los hallazgos y la línea del trabajo, puede contribuir a la disminución del FA y promulgar por un saber legítimo y claro: es necesario un espacio de reflexión que visualice el fenómeno y ponga a conversar a los diferentes actores, con miras lograr una verdadera concientización de lo nocivo del fenómeno. Es importante hablar del fenómeno, hacerlo visible, escuchar a los estudiantes y planear con ellos actividades intervencionistas como campañas de sensibilización. El objetivo es que se forme en ellos un mandato propio, no forzado ni punitivo, que les estimule un cambio de actitud, como describe Camps (2007).

Así las cosas, se requiere de un trabajo trasversal, permanente, tendiente a lograr que los estudiantes se rijan bajo un código de honor donde los valores éticos y morales sean resaltados y prevalezcan sobre lo práctico e inmediato. Estas determinaciones deben nacer de los mismos estudiantes mediante la reflexión y sin presiones, constituyéndose así en un 
mandato autónomo que garantice una real incorporación de los valores éticos que queremos rescatar.

Esta tarea ha de ser conjunta con los docentes ya que serán ellos, en últimas, los garantes del proceso. Es por esto por lo que la sensibilización de los profesores a los aspectos concernientes con el FA se hace necesaria para lograr el impacto esperado, debe haber una sincronía entre la concepción del fenómeno por parte de la administración, los estudiantes y los profesores.

\section{Trabajo conjunto con organismos internacionales}

El Instituto Internacional para el Planeamiento de la Educación (IIPE), es un organismo creado por la Organización de las Naciones Unidas para la Educación, la Ciencia y la Cultura (UNESCO), que ha desarrollado un conocimiento específico sobre los sistemas educativos de América Latina, para permitir la toma de decisiones con una visión actualizada y contextualizada sobre temas trascendentales en el planeamiento, la gestión y la evaluación de políticas educativas. Dentro de sus líneas de trabajo existen capítulos dedicados al asunto que nos ocupa del fraude académico, por tales motivos, puede ser una fuente valiosa de consulta y de trabajo conjunto con la universidad para la adecuada intervención del fenómeno.

\section{Referencias}

Agud, J. (2014). Fraude y plagio en la carrera y en la profesión. Revista Clínica Española, 214(7), 410-414.

$\begin{array}{llll}\text { Arancibia, M. } & \text { D. } & \text { (2010). Pensamiento }\end{array}$ complejo. https://www.philosophica.info/archivo/2010/voces/pensamiento_comple jo/Pensamiento_Complejo.html

Ayala-Gaytán, E. A., \& Quintanilla-Domínguez, C. M. (2014). Actitudes y causas de fraude entre estudiantes universitarios en México: un estudio exploratorio. Magis. Revista Internacional de Investigación en Educación, 6(13), 17-30.

Beasley, E. M. (2016). Comparing the demographics of students reported for academic dishonesty to those of the overall student population. Ethics \& Behavior, 26(1), 4562.

Beléndez-Vázquez, M., Comas-Forgas, R., Martín-Llaguno, M., Muñoz-González, A. \& TopaCantisano, G. (2011). Plagio y otras prácticas académicamente incorrectas entre el alumnado universitario de nuevo ingreso. IX Jornadas Redes de invesfigación en docencia universitaria, 16-17 junio 2011. 
Blumer, H. y Alonso, P. R. (1982). El interaccionismo simbólico: perspectiva y método. Hora Barcelona.

Bunn, D. N., Caudill, S. B. \& Gropper, D. M. (1992). Crime in the classroom: An economic analysis of undergraduate student cheating behavior. The Journal of Economic Education, 23(3), 197-207.

Camps, V. (2007). La excelencia en las profesiones sanitarias. Revista HumanitasHumanidades Médicas,

(21), 1-13. http://www.ehu.eus/SEMDE/archivos_pdf/archivos_pdf/Victoria\%20Camps\%20La \%20excelencia\%20en\%20las\%20profesiones\%20sanitarias-1.pdf

Camps, V. (2012). El gobierno de las emociones. Herder Editorial.

Castro, A. M. P., Espiñeira-Bellón, E.-M., Losada-Puente, L. \& Pérez, E. M. G. (2019). El alumnado universitario ante políticas institucionales y de aula sobre plagio. Bordón. Revista de pedagogía, 71(2), 139-153.

Ceballos-Villada, Z. R. (2011). Comportamientos, pensamientos y sentimientos frente al fraude académico en ámbitos universitarios: Institución universitaria CESMAG Universidad Mariana.

Chipombela, A., Pereira, A. M. A. d. F. \& Calivangue, E. A. M. (2020). Fraude académico en la universidad. Medidas desde la Universidad Cuito Cuanavale. Revista Cubana de Educación Superior, 39(2), 1-12. http://scielo.sld.cu/scielo.php?script=sci_abstract\&pid=S025743142020000200005\&lng=es\&nrm=iso

Comas, R., Sureda, J., Casero, A. \& Morey, M. (2011). La integridad académica entre el alumnado universitario español. Estudios pedagógicos (Valdivia), 3オ1), 207-225.

Cronan, T. P., Mullins, J. K., \& Douglas, D. E. (2015) Further Understanding Factors that Explain Freshman Business Students' Academic Integrity Intention and Behavior: Plagiarism and Sharing Homework. Journal of Business Ethics, 147(1), 197-220. https://doi.org/10.1007/s10551-015-2988-3

Duque, L. F., Toro, J. A. y Montoya, N. (2010). Tolerancia al quebrantamiento de la norma en el área metropolitana de Medellín, Colombia. Opinião Pública, 16(1), 65-89.

Eckstein, M. A. (2003). Combating academic fraud: Towards a culture of integrity. International Institute for Educational Planning. 
Fernández, J. y Ordóñez, C. (2004). El fraude académico en la Universidad de los Andes ¿qué?, ¿qué tanto? Y ¿por qué?. Revista de Estudios Sociales, (18), 369-375.

Gama, P., Peixoto, P., Seixas, A. M., Almeida, F. y Esteves, D. (2013). A ética dos alunos de administração e de economia no ensino superior. Revista de Administração Contemporânea, (17), 620-641.

Giménez, R. C. (2007). Aplicación de la teoría fundamentada (grounded theory) al estudio del proceso de creación de empresas. Decisiones basadas en el conocimiento y en el papel social de la empresa. Decisiones Globales, (1), 1-13.

Gutiérrez, M., Ball, M. y Márquez, E. (2008). Signo, significado e intersubjetividad: una mirada cultural. Educere, 12(43), 689-695.

Hernández, L. N. (2013). Comunicación y educación bajo la impronta del pensamiento complejo. Razón y palabra, (83), 320-330.

Martinez Gomez, A., Borjas, M. P. y Andrade, J. J. (2015). El fraude académico universitario: el caso de una universidad privada en la ciudad de Barranquilla. Zona Próxima, (23).

Martinez, L. \& Ramírez, R. (2018). Fraude académico en universitarios en Colombia: ¿Qué tan crónica es la enfermedad? Educação e Pesquisa, (44), 1-19. http://dx.doi.org/10.1590/S1517-9702201706157079

Mejía-Quintana, Ó. (2018). Cultura política y justicia mafiosa en Colombia. Dialogos de Saberes, (48), 91-108. https://doi.org/10.18041/0124-0021/dia-logos.48.2018.4715

Mejía-Sabogal, D., Pineda, A., López, Á., Gómez, T. y Nieves, L. (2019). Vida universitaria: una visión desde las vivencias y prácticas del fraude académico. Revista de la Universidad de La Salle, (80), 181-203.

Mendoza, P. A. A., Ramos, Y. L. M., Ortiz, Ó. E. C. y Jaramillo, J. M. (2010). Comprensión del significado desde Vygotsky, Bruner y Gergen. Diversitas, 6(1), 37-49.

Mora, M. G. (2015). ¿Por qué fracasa Colombia?: Un viaje a través de la historia nacional. Editorial Porthos.

Muñoz-Fonnegra, S. (2010). La elección ética: Sobre la crítica de Kierkegaard a la filosofía moral de Kant. Estudios de Filosofía, (41), 81-109.

Ordóñez, C. L., Mejía, J. F. y Castellanos, S. (2006). Percepciones estudiantiles sobre el fraude académico: hallazgos y reflexiones pedagógicas. Revista de Estudios Sociales, (23), 37-44. 
Pardo, A. y Rodríguez-Casals, C. (2019). Fraude académico en la Universidad: análisis de un caso real, el conflicto y su resolución. Archivos analíticos de políticas educativas, 27(40), 1-16. https://dialnet.unirioja.es/servlet/articulo?codigo=7434525

Ramírez, A. S. (2019). El plagio: un dolor de cabeza para estudiantes y académicos. En E. Serna (Ed.), Revolución en la formación y la capacitación para el siglo XXI (pp. 373376). Medellín, Antioquia, Instituto Antioqueño de Investigación.

Shen, Q., Teo, M., Winter, E., Hart, E., Chew, S. H. \& Ebstein, R. P. (2016). To cheat or not to cheat: Tryptophan hydroxylase 2 SNP variants contribute to dishonest behavior. Frontiers in behavioral neuroscience, 10(82), 1-9. https://www.frontiersin.org/articles/10.3389/fnbeh.2016.00082/full

Strauss, A. L. \& Corbin, J. (2002). Bases de la investigación cualitativa: técnicas y procedimientos para desarrollar la teoría fundamentada. Universidad de Antioquia Medellín.

Sureda-Negre, J., Cerdá-Navarro, A., Calvo-Sastre, A. \& Forgas, R. C. (2020). Las conductas fraudulentas del alumnado universitario español en las evaluaciones: valoración de su gravedad y propuestas de sanciones a partir de un panel de expertos. Revista de Investigación Educativa, 38(1), 201-219.

Sureda-Negre, J., Comas-Forgas, R. y Oliver-Trobat, M. F. (2015). Plagio académico entre alumnado de secundaria y bachillerato: Diferencias en cuanto al género y la procrastinación. Comunicar: Revista Científica de Comunicación y Educación, 22(44), 103-111.

Suárez, J. C. P. (2021). El interaccionismo simbólico: oportunidades de investigación en el aula de clase. Revista Internacional De Pedagogía E Innovación Educativa, 1(1), 211-228.

Teodorescu, D., Andrei, T., Tusa, E., Herteliu, C. \& Stancu, S. (2007). Analyzing the Students' Academic Integrity using Quantitative Methods. Journal of Applied Quantitative Methods, 2(2), 211-220.

Veludo-de-Oliveira, T. M., Aguiar, F. H. O. d., Queiroz, J. P. d. \& Barrichello, A. (2014). Cola, plágio e outras práticas acadêmicas desonestas: um estudo quantitativo-descritivo sobre o comportamento de alunos de graduação e pós-graduação da área de negócios. RAM. Revista de Administração Mackenzie, 15(1), 73-97.

Vich, V. (2019). Desculturalizar la cultura: la gestión cultural como forma de acción política. Siglo XXI Editores. 
Wiesenfeld, K. y Jaramillo, L. G. (2011). Cómo se consiguen las buenas calificaciones. Revista Universidad EAFIT, 45(156), 104-106. 